\title{
Evaluación de la conducción eficiente en un motor de encendido provocado, a $2810 \mathrm{msnm}$
}

\author{
Julio C. Leguísamo(1), Edilberto A. Llanes-Cedeño(1), Santiago F. Celi-Ortega(1), y Juan C. Rocha-Hoyos ${ }^{(1,2)}$ \\ (1) Universidad Internacional SEK, Fac. de Arquitectura e Ingenierías, Grupo de Investigación Eficiencia, Impacto \\ Ambiental e Innovación en la Industria y el Transporte, Quito-Ecuador (correo-e: julio.leguisamo@uisek.edu.ec; \\ carlos.rocha@uisek.edu.ec; antonio.llanes@uisek.edu.ec) \\ (2) Escuela Superior Politécnica de Chimborazo, Facultad de Mecánica, Carrera de Ingeniería Automotriz, Riobamba- \\ Ecuador (correo-e: juan.c.rocha1@gmail.com)
}

Recibido Abr. 1, 2019; Aceptado May. 28, 2019; Versión final Ago. 8, 2019, Publicado Feb. 2020

\section{Resumen}

En el presente trabajo, se caracteriza la aplicación de la conducción eficiente con respecto a la conducción normal para la evaluación de las emisiones contaminantes y de la economía de combustible de un motor de combustión interna de encendido provocado, a $2810 \mathrm{msnm}$. Como estudio de caso se utilizó un vehículo de cilindraje $1498 \mathrm{~cm}^{3}$ de inyección indirecta a gasolina. Se realizaron las pruebas en un dinamómetro de chasis aplicando el ciclo Japonés Modo 10-15. Mediante la experimentación se obtuvo el consumo específico de combustible y las concentraciones de $\mathrm{CO}, \mathrm{HC}$ y NOx. Con estos datos de emisión se calcularon los factores de cada gas contaminante. Los resultados obtenidos revelan que el consumo de combustible y el factor de emisión de óxidos nitrosos (NOx) se reducen en un $19.8 \%$ y en un $10 \%$ respectivamente al aplicar una conducción eficiente en relación a una conducción normal. Para el caso del factor de emisión de monóxido de carbono $\mathrm{CO}$ y $\mathrm{HC}$ las diferencias no son significativas.

Palabras clave: conducción ecológica; consumo de combustible; emisiones; motor de encendido provocado; condiciones de altitud

\section{Efficient driving evaluation an ignition engine at $\mathbf{2 8 1 0}$ meters above sea level}

\begin{abstract}
In the present work, is characterized the application of efficient driving with respect to normal driving for the evaluation of polluting emissions and the fuel economy of internal ignition combustion engine at 2810 masl, as case study used a $1498 \mathrm{~cm} 3$ cylinder vehicle with indirect gasoline injection. The tests were performed on a chassis dynamometer applying the cycle Japanese Mode 10-15; through experimentation, the specific fuel consumption and the concentrations of $\mathrm{CO}, \mathrm{HC}$ and $\mathrm{NOx}$ were obtained, with these emission data the factors of each polluting gas were calculated. The results obtained reveal that the fuel consumption and the emission factor of nitrous oxides (NOx) are reduced by $19.8 \%$ and by $10 \%$ respectively when applying efficient driving in relation to normal driving. In the case of the carbon monoxide emission factor $\mathrm{CO}$ and $\mathrm{HC}$, the differences are not significant.
\end{abstract}




\section{INTRODUCCIÓN}

Uno de los focos de contaminación del aire más importante en la actualidad son los vehículos de combustión (Rogula, 2008; Rocha et al., 2018). De hecho, diversos estudios sitúan a las partículas emitidas por los vehículos como una causa de mortalidad de la población mundial (Kinney, 2000), además son los causantes de producir el $30 \%$ de emisiones de gases de efecto invernadero en el mundo (Krajzewicz et al., 2014). Por esto la importancia de realizar estudios para determinar la reducción de consumo de combustible y emisiones contaminantes. Existen varios métodos para reducir la demanda de combustible y las emisiones contaminante, basados en la mejor eficiencia de los motores: uso de biocombustibles, mejoras aerodinámicas y reducción de peso con nuevos materiales (Magaña, 2014; Vega et al., 2018). Hay otro método conocido como conducción eficiente, ecodriving, conducción ecológica o manejo ecológico, que es una técnica de conducción basada en el control por parte del conductor de la velocidad, la marcha y la desaceleración (Yanzhi et al., 2017) este estilo de conducción aumenta la seguridad, reduce el consumo de combustible y las emisiones contaminantes (Baric et al., 2013).

De acuerdo con Alam y McNabola (2014) muchos países han adoptado políticas de conducción ecológica dentro del sector del transporte, en un intento de reducir el consumo de energía y emisiones de contaminantes. Estudios realizados han evaluado los beneficios de la conducción ecológica a través de la experimentación en pruebas dinámicas y estáticas (Huang et al., 2018). Barth y Boriboonsomsin (2009) en su estudio al aplicar los consejos conducción eficiente, determinaron un ahorro de combustible en pruebas en ruta del $13 \%$ y en pruebas dinámicas de $37 \%$; mientras que la reducción de $\mathrm{CO}_{2}$ fue de $12 \%$ en ruta y $35 \%$ en estática. Mensing et al., (2014) demostraron la existencia de una diferencia entre un ciclo económico y un ciclo ecológico, con una reducción de $0.2 \mathrm{~L} / 100 \mathrm{~km}$ en consumo de combustible, $5.1 \%$ de $\mathrm{CO}_{2}$ y $38.1 \%$ de NOX. En el estudio realizado por Ho et al., (2015) después de realizar de 30 a 45 sesiones de entrenamiento los conductores de prueba redujeron las emisiones de carbono y ahorro de combustible a más del $10 \%$ con un modelo tendencial exponencial. En otro estudio sobre la efectividad de los cursos de eco-driving para vehículos ligeros se determinó que con un entrenamiento de cuatro horas a 10 conductores durante un mes se ahorró un $5.8 \%$ de combustible (Beusen et al., 2009). Mientras que Qiang, y Chung (2011), determinaron que en escenarios de congestión de tráfico el desempeño ambiental es negativo, aunque se ahorra un $11 \%$ de combustible sin mayor aumento en el tiempo de viaje.

En la ciudad colombiana de Pereira ubicada en una región montañosa a 1441 metros sobre el nivel del mar se implementó la metodología de pruebas basada en la Regulación CFR 40 de los Estados Unidos y empleando conducción eficiente se identificó un aumento en el rendimiento del $15 \%$ y $20 \%$ en pruebas de laboratorio y ruta respectivamente (Castillo et al. 2019). Mientras que Rodríguez et al., (2016) en un estudio en Bogotá se determinó que al aplicar ecodriving las emisiones anuales se reducirían en un $12 \%$ para $\mathrm{CO}_{2}$, $13 \%$ para $\mathrm{CO}$ y $\mathrm{HC}$, y $24 \%$ para $\mathrm{NO}_{x}$, lo cual representa un posible ahorro en el consumo de combustible de entre 35 y 85 millones de galones por año y los beneficios económicos potenciales totales de hasta 1400 millones de dólares por año.

Los EE.UU, la Unión Europea y Japón han desarrollado métodos propios para la verificación de certificación y control de campo de gases de escape y consumo de combustible de los vehículos que se comercializan en estas zonas. Otros países han adoptado estos métodos de igual forma o también de forma modificada lo cual ha dado lugar a las normas de emisión de cada país (Xuekai et al., 2016). Las normas determinan distintos ciclos de ensayo, derivados de registros reales o construidos en base a aceleración y velocidad constantes, para determinar las masas de sustancias nocivas expulsadas y el consumo de combustible en un recorrido fijo. Datos con los cuales se puede desarrollar inventarios de emisiones para generar políticas de prevención y de control con respecto a la salud, medio ambiente, industria, etc. (Binder et al., 2014).

El estudio realizado por Lapuerta et al., (2006) indica que, en condiciones sobre los $2000 \mathrm{msnm}$ los motores de combustión interna tienen menor eficiencia con respecto a consumo de combustible y concentración de emisiones contaminantes. A partir de lo anteriormente expuesto el presente estudio tuvo como objetivo caracterizar el consumo de combustible y las concentraciones de emisión de gases contaminantes de un motor de combustión interna MEP a 2810 msnm, por medio de un modo de conducción normal y conducción eficiente, para determinar si existen diferencias significativas con respecto a los factores de emisión de gases.

\section{MATERIALES Y MÉTODOS}

En el presente estudio se evalúa el consumo de combustible y los factores de emisión de un vehículo al realizar una prueba en un dinamómetro, de un ciclo de prueba internacional que se asemeje a las condiciones de tráfico de la ciudad de Quito. Determinando dos variables de estudio la primera consiste en una conducción normal y la segunda está determinada por una conducción ecodriving, ejecutadas por el mismo conductor. 


\section{Conducción normal y conducción ecológica}

La conducción normal del vehículo se denomina a la manera de conducir habitualmente durante un recorrido, mientras que la conducción ecológica consiste en una serie de normas que debe aplicar el conductor al conducir el mismo recorrido. En este caso el conductor realizará primero las pruebas de conducción normal y después de realizarlas recibirá un curso de capacitación de 8 horas de conducción ecológica.

Las instrucciones que se deben seguir para aplicar conducción eficiente son: los cambios de marcha deben hacerse entre 2000 y 2500 rpm, circular en marchas largas y a bajas revoluciones, usar la primera marcha sólo para poner en movimiento el vehículo, evitar acelerones y frenazos, desacelerar con el freno motor, detener el auto utilizando sólo el freno y no utilizar el punto muerto cuando se frena, aprovechar la inercia del coche siempre que sea posible, en las paradas no escalar todas las marchas sino mantener la marcha puesta hasta pisar embrague y detenerse y por último descender las pendientes en marcha.

\section{Caracterización de emisiones contaminantes y consumo de combustible}

La investigación se desarrolló durante una simulación del ciclo de ensayo modo 10-15 de la legislación japonesa (Qu et al., 2015; Liu, y Frey, 2015) enel banco de pruebas de chasis. Debido a que este ciclo en relación a los ciclos UE/ECE de la legislación europea y el ciclo FTP 75 de la legislación americana presenta velocidades medias y máximas mayores a las que se producen en la realidad en la ciudad, las que fueron determinadas en el estudio de Quichimbla (2017), el cual se observa en la tabla 1 donde el comportamiento de marcha del ciclo es similar al comportamiento que se obtuvo en Quito, la cual no posee un ciclo de prueba validado. Este ciclo se realiza con el vehículo en temperatura de funcionamiento, tiene una longitud de 4,16 km, una duración de 660 segundos y las características del ciclo de la velocidad en función del tiempo se indica en la figura 1 (Barlow et al., 2009).

Tabla1: Características del vehículo de prueba

\begin{tabular}{|l|l|l|l|l|}
\hline & UE/ECE & FTP 75 & MODO 10-15 & ESTUDIO EXPERIMENTAL \\
\hline Velocidad Media & $32.5 \mathrm{~km} / \mathrm{h}$ & $34,1 \mathrm{~km} / \mathrm{h}$ & $22,7 \mathrm{~km} / \mathrm{h}$ & $19,18 \mathrm{~km} / \mathrm{h}$ \\
\hline Velocidad Máxima & $120 \mathrm{~km} / \mathrm{h}$ & $91,2 \mathrm{~km} / \mathrm{h}$ & $70 \mathrm{~km} / \mathrm{h}$ & $78,32 \mathrm{~km} / \mathrm{h}$ \\
\hline
\end{tabular}

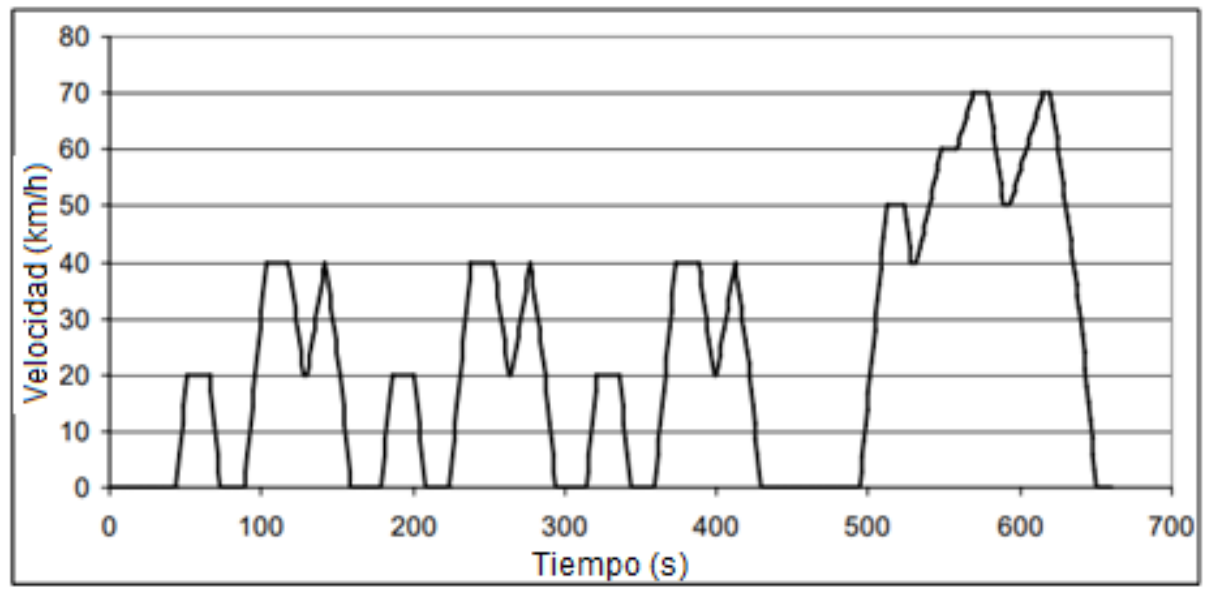

Fig. 1: Ciclo de conducción Japón Modo 10-15 ensayo para turismo y vehículos industriales ligeros

Los valores que se determinan experimentalmente para el cálculo de los factores de emisión son las concentraciones de volumen de $\mathrm{NO}, \mathrm{HC}, \mathrm{CO}$ y $\mathrm{CO}_{2}$ y el consumo de combustible. Los equipos de análisis de emisiones contaminantes miden la concentración volumétrica expresada en porcentaje (\%). El análisis dinámico del comportamiento de los gases de escape en los vehículos se expresa en gramos por kilómetro (g/km), para ello se utiliza el modelo simplificado de combustión el cual representa la conversión de la mezcla airecombustible en sus principales productos según la ecuación 1, donde las variables $a, b, c, d$, e, f y m son coeficientes estequiométricos desconocidos y definidos en moles formados por mol de combustible consumido. En vista que el $\mathrm{CO}_{2}, \mathrm{CO}$ y $\mathrm{HC}$ son resultados de la combustión que contienen carbono, es posible desarrollar un balance de masa respecto a la cantidad de carbono presente en el combustible y en estos 3 productos de la combustión. Los productos se consideran como base para el análisis de las emisiones y las 
características medias de los combustibles, por tanto, del balance de masa para el carbono se puede escribir como la ecuación 1 y 2 (Frey y Eichenberger, 1997).

$$
\begin{aligned}
& C H y+m\left(0.21 O_{2}+0.79 N_{2}\right) a C O+b H_{2} O+c C_{3} H_{6}+d C_{2}+e N_{2}+f N O \\
& 1=a+3 c+d
\end{aligned}
$$

Los contaminantes genéricos son medidos en el tubo de escape y se analizan según la ecuación 3 , donde: Rco es la relación de $\mathrm{CO}$ respecto a $\mathrm{CO}_{2}$ en porcentaje y $\mathrm{RHC}$ es la relación de $\mathrm{HC}$ respecto a $\mathrm{CO}_{2}$ en porcentaje. El equipo de medición de gases entrega datos segundo a segundo sobre una base de volumen de todos los resultados de la combustión, y aplicando las relaciones anteriores se obtiene la porción de $\mathrm{CO}_{2}$.

$$
d=\frac{1}{R_{C O}+3 R_{H C}+1}
$$

Mediante la ecuación 4 se determinan los factores de emisión $\left(F E_{i}\right)$ en gramos de contaminante por kilómetro recorrido, donde el subíndice $i$ se relaciona con los contaminantes $\mathrm{CO}, \mathrm{HC}$ y NOx, $M W_{\text {comb }}$ al peso molecular equivalente del combustible, $M W_{i}$ al peso molecular equivalente de los contaminantes, $\rho_{\text {comb }}$ es la densidad del combustible en $\mathrm{g} / \mathrm{m}^{3}$ y c.c. es el consumo de combustible por distancia recorrida en $\mathrm{m}^{3} / \mathrm{km}$ (Frey y Eichenberger, 1997).

$$
F E_{i}=\frac{R_{i}}{R_{C O}+3 R_{H C}+1} \times \frac{M W_{i}}{M W_{c o m b}} x \rho_{\text {comb }} x \text { c.c. } ;[\mathrm{g} / \mathrm{km}]
$$

\section{Equipos utilizados en las evaluaciones}

Se seleccionó el automóvil más vendido en la ciudad de Quito de acuerdo a los informes anuales de ventas de vehículos de la Asociación de Empresas Automotrices del Ecuador. El cual desde el 2009 año en que se empezó a comercializar este vehículo hasta el 2018 se han reportado un total de 24994 unidades y esta tendencia se mantiene en todo el país. El vehículo es un Chevrolet Aveo family cuyas características se describen en la tabla 2. Previo a las pruebas experimentales en el auto se realizó un mantenimiento preventivo, el cual consistió en el cambio de aceite de motor, filtro de aire, filtro de combustible, bujías, limpieza de inyectores, verificación del estado de cables de bujías, revisión de sensores y actuadores.

Tabla2: Características del vehículo de prueba

\begin{tabular}{|l|l|}
\hline Cilindrada & $1498 \mathrm{~cm}^{3}$ \\
\hline Relación de compresión & $9.5: 1$ \\
\hline Diámetro x carrera & $76.5 \mathrm{~mm} \times 81.5 \mathrm{~mm}$ \\
\hline Potencia & $83 / 5600 \mathrm{hp} / \mathrm{rpm}$ \\
\hline Par motor & $127 / 3000 \mathrm{Nm} / \mathrm{rpm}$ \\
\hline Válvulas & $8 \mathrm{SOHC}$ \\
\hline Sistema de inyección de & MPFI \\
\hline combustible & \\
\hline Catalizador de gases de escape & Tres vías \\
\hline Sistema de Encendido & Chispa perdida DIS \\
\hline Motor- tracción & Delantera \\
\hline Transmisión & Manual 5 velocidades \\
\hline Tanque de combustible & $45 \mathrm{~L}$ \\
\hline Kilometraje & $36344 \mathrm{~km}$ \\
\hline
\end{tabular}

Para la simulación del ciclo de prueba se utilizó el dinamómetro de chasis MAHA LPS 300 el que permite simular un perfil de velocidad en función del tiempo para realizar pruebas dinámicas en vehículos a diésel y gasolina. El mismo permite cargar diferentes ciclos de ensayo de las distintas legislaciones o ciclos autodiseñados, para poder determinar exactamente las cantidades de sustancias nocivas expulsadas por un vehículo, ya que no se toma en consideración el tráfico; además permite realizar comparativa entre diferentes ciclos de conducción.

La medida de la concentración de volumen de los gases de $\mathrm{CO}, \mathrm{CO}_{2}, \mathrm{O}_{2}$; la ppm de $\mathrm{HC}$ y $\mathrm{NOx}$ se realizó con un analizador de gases MAHA GT5, estos parámetros permiten obtener los factores de emisión de los gases contaminantes para determinar la diferencia que se produce al aplicar las diferentes variables de prueba. Los datos técnicos del equipo se indican en la tabla 3. 
Tabla 3: Datos técnicos del analizador MAHA GT5.

\begin{tabular}{|l|c|c|c|c|}
\hline Gases Analizables & $\mathrm{CO}$ (Vol. \%) & $\mathrm{CO}_{2}$ (Vol. \%) & $\mathrm{HC}$ (ppm) & $\mathrm{O}_{2}$ (Vol. \%) \\
\hline Rango de medición & $0-15.00$ & $0-20.0$ & $\begin{array}{l}0-2000 \text { (Hexano) } \\
0-4000 \text { (Propano) }\end{array}$ & $0-25.00$ \\
\hline Precisión de medida & 0.06 & 0.5 & 12 & 0.01 \\
\hline Resolución valores de medida & 0.001 & 0.01 & 0.1 & 0.01 \\
\hline Principio de medida & Infrarrojo & Infrarrojo & Infrarrojo & Electro-químico \\
\hline
\end{tabular}

La medida de la cantidad de combustible consumida se la realiza con un tanque de presión de combustible externo con una capacidad de 5 litros, que se puede presurizar hasta 80 psi; mediante el cual se abastece de combustible a presión al vehículo, y se obtiene la cantidad de combustible consumida en el ciclo. El tanque de presión de combustible consta de una bomba manual con el cual se presuriza el sistema, para garantizar el flujo constante de combustible, su presión es revisada en cada prueba (Rocha et al., 2018).

Se utiliza un escáner automotriz que cumple la legislación OBDIl que prescribe una estandarización de las informaciones de lectura en la línea de datos conforme a las especificaciones de la SAE J 1979, 2002. Se usa el scanner G-Scan2, que es un equipo genérico con el cual se observó los valores de trabajo de los sensores, actuadores e interruptores. En ese caso se selecciona la línea de rpm para verificar el correcto rango de funcionamiento al aplicar ecodriving entre 2000 y 2500 rpm.

\section{Protocolo de pruebas}

Se realizaron cinco pruebas del ciclo para cada tipo de conducción a 2810 metros de altura sobre el nivel del mar, una temperatura ambiente de $14^{\circ} \mathrm{C}$ y utilizando gasolina extra con una densidad de $744 \mathrm{~kg} / \mathrm{m}^{3}$ en la ciudad de Quito-Ecuador. Esta prueba se ejecuta en los laboratorios del Centro de Transferencia Tecnológica para la Capacitación e Investigación en Control de Emisiones Vehiculares (CCICEV); mediante este ciclo dinámico se determinan las concentraciones volumétricas de $\mathrm{CO}, \mathrm{CO}_{2}, \mathrm{HC}$ y NOx cada 0.1 segundos por el lapso total de duración del ciclo de 660 segundos y el consumo de combustible. Para la realización de las pruebas se sigue el protocolo que se describe en los siguientes puntos: (1) Verificar que el sistema de escape del vehículo no tenga perforaciones ni fugas; (2) Verificar que la presión de los neumáticos sea la recomendada por el fabricante del vehículo. (3) Anclar el vehículo a los soportes del dinamómetro. (4) Instalar y asegurar el equipo para la medición de gases MAHA MGT5, medición de consumo de combustible y control de rpm; (5) Encender el vehículo y calentar el motor hasta que alcance la temperatura de operación normal que corresponde a $95 \stackrel{\circ}{\circ} \mathrm{C}$. (6) Revisar que accesorios del vehículo estén desactivados. (7) Instalar el ventilador del dinamómetro; (8) Iniciar y seguir el ciclo modo 10-15 cargado en el dinamómetro;(9) Grabar los datos obtenidos en el computador; (10) Repetir el procedimiento para cada prueba. En el dinamómetro MAHA LPS 300 , se sometió a la prueba dinámica del ciclo japonés modo 10-15. Adicionalmente en la parte posterior del sistema de escape, se encuentra conectado el analizador de gases MAHA GT5 y el canister para garantizar el flujo constante de combustible. En la figura 2 se observa el vehículo preparándose para una prueba.

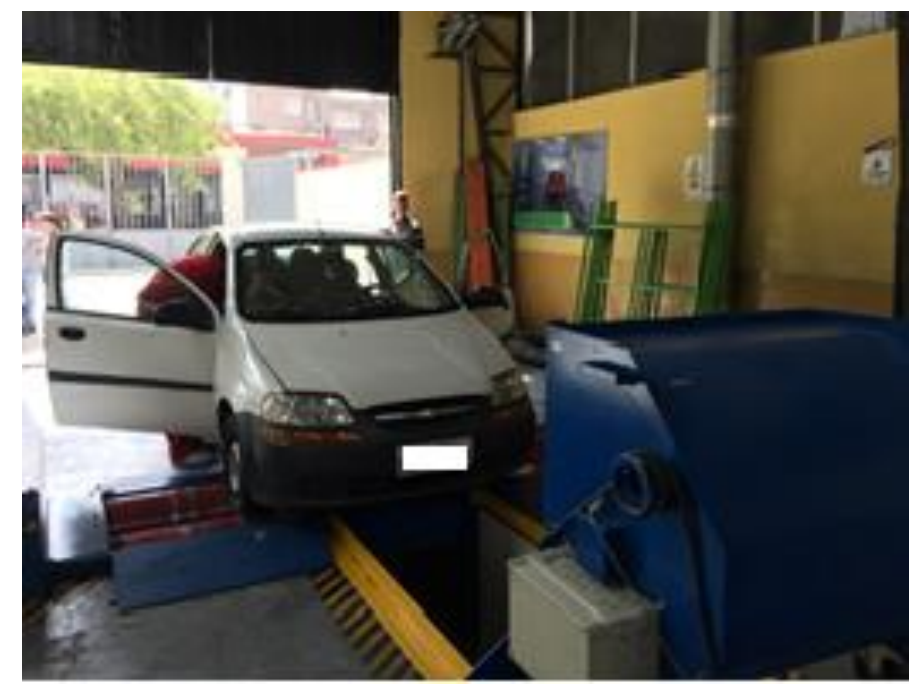

Fig.2: Vehículo preparándose para prueba en dinamómetro 
Posteriormente se realizó el control de rangos (Tipanluisa et al., 2018) para determinar si los datos obtenidos en la experimentación son fiables debido a que pueden presentarse desviaciones con respecto al objetivo específico y se pueden manifestar en forma de datos inexactos provocando una excesiva variabilidad respecto a los valores deseables. En el estudio se presenta el comportamiento de las variables dependientes: consumo de combustible $(\mathrm{L} / \mathrm{km})$ y factores de emisiones de gases producto de la combustión (FCO, FHC y FNOx), en función de la variable independiente modo de conducción (conducción eficiente identificada por 2 y conducción normal por 1). Para el tratamiento estadístico de los resultados se toma en consideración las propuestas de varios autores como la abordada por Guaman et al., (2019). Para determinar si entre los grupos experimentales existe diferencia significativa, se usó el análisis ANOVA con el software STATGRAPHICS Centurion XVI, aplicándose pruebas de comparación múltiple de medias para este caso la LSD (Least Significant Difference) en un $95 \%$ de confianza (Kolanjiappan, 2017; Guardia et al., 2018).

\section{RESULTADOS Y DISCUSIÓN}

Una vez realizadas todas las pruebas requeridas, se procesan los datos y se presenta los siguientes resultados debidamente ajustados. En la Tabla 4, se presentan los datos de las pruebas realizadas en el Laboratorio del CCICEV del ciclo Japonés Modo $10-15$ y el cálculo de los factores de emisión de cada los gases contaminantes.

Tabla 4: Consumo de combustible y Porcentaje de concentración de emisiones contaminantes

\begin{tabular}{|c|c|c|c|c|c|c|c|c|c|c|c|c|c|c|}
\hline \multirow[t]{2}{*}{ Prueba } & \multicolumn{7}{|c|}{ Conducción Normal (1) } & \multicolumn{7}{|c|}{ Conducción Ecológica (2) } \\
\hline & \begin{tabular}{|c|} 
Consumo \\
$(\mathrm{L} / 4.16 \mathrm{~km})$
\end{tabular} & \begin{tabular}{|c|}
$\mathrm{CO}$ \\
$(\% \mathrm{v})$
\end{tabular} & $\begin{array}{l}\mathrm{HC} \\
(\% \mathrm{v})\end{array}$ & $\begin{array}{r}\text { NOX } \\
(\% \mathrm{~V})\end{array}$ & $\begin{array}{l}\text { FCO } \\
(\mathrm{g} / \mathrm{km})\end{array}$ & $\begin{array}{l}\mathrm{FHC} \\
(\mathrm{g} / \mathrm{km})\end{array}$ & $\begin{array}{l}\text { FNOX } \\
(\mathrm{g} / \mathrm{km})\end{array}$ & \begin{tabular}{|c|} 
Consumo \\
$(\mathrm{L} / 4.16 \mathrm{~km})$
\end{tabular} & $\begin{array}{l}\mathrm{CO} \\
(\% \mathrm{v})\end{array}$ & $\begin{array}{l}\mathrm{HC} \\
(\% \mathrm{v})\end{array}$ & $\begin{array}{r}\text { NOX } \\
(\% \mathrm{v})\end{array}$ & $\begin{array}{l}\text { FCO } \\
(\mathrm{g} / \mathrm{km})\end{array}$ & $\begin{array}{l}\mathrm{FHC} \\
(\mathrm{g} / \mathrm{km})\end{array}$ & $\begin{array}{l}\text { FNOX } \\
(\mathrm{g} / \mathrm{km})\end{array}$ \\
\hline 1 & 55 & 0.23 & 0.007 & 0.042 & 2.23 & 0.096 & 0.433 & & 0.348 & 0.006 & 0.046 & 2.71 & 0.067 & 0.385 \\
\hline 2 & 0.56 & 0.27 & 0.007 & 0.040 & 2.30 & 0.074 & 0.419 & 0.45 & 0.332 & 0.006 & 0.046 & 2.61 & 0.068 & 0.384 \\
\hline 3 & 0.54 & 0.22 & 0.005 & 0.046 & 2.22 & 0.072 & 0.479 & 0.44 & 0.356 & 0.006 & 0.052 & 2.69 & 0.064 & 0.401 \\
\hline 4 & 0.53 & 0.29 & 0.004 & 0.040 & 2.79 & 0.058 & 0.407 & 0.42 & 0.388 & 0.006 & 0.051 & 2.84 & 0.069 & 0.403 \\
\hline 5 & 0.54 & 0.26 & 0.005 & 0.043 & 2.48 & 0.071 & 0.434 & 0.42 & 0.365 & 0.006 & 0.051 & 2.63 & 0.062 & 0.396 \\
\hline Promedio & 0.544 & 0.26 & 0.005 & 0.042 & 2.40 & 0.074 & 0.434 & 0.436 & 0.357 & 0.006 & 0.049 & 2.70 & 0.066 & 0.394 \\
\hline
\end{tabular}

En el análisis de resultados se debe considerar que la altura influye directamente en la combustión debido a que se reduce la cantidad de oxígeno en la mezcla y bajo estas condiciones, es menos eficiente y de esta manera se influye en las emisiones y el consumo de combustible (Rocha-Hoyos et al., 2019). La Tabla 5 presenta el ANOVA, donde se puede destacar que el Valor-p muestra diferencia significativa entre los grupos. La Tabla 6 y la Figura 3 representa la prueba de múltiple rango y gráfico de caja y bigotes para la variable dependiente Consumo de combustible, en la misma se aplica el procedimiento de diferencia mínima significativa (LSD) de Fisher con un nivel del 95.0\% de confianza. Se muestra que existe diferencia significativa entre los modos de conducción, siendo la conducción eficiente la de mejor resultado. Se obtiene, además, que el consumo de combustible al aplicar el modo de conducción ecológica en 0.436 litro de combustible se puede recorrer $4.16 \mathrm{~km}$, en comparación con la conducción normal que para recorrer el mismo kilometraje se debe consumir 0.544 litros, lo cual representa una diferencia del $19.8 \%$; estos resultados son similares con el estudio de Baric et al., (2013) en los cuales el consumo de combustible del conductor antes del entrenamiento ascendió a 27,64 L/100km y después de la formación a 20,9 L/100 km que significa una reducción del $24.38 \%$. Además, coincide con lo referenciado por Luján et al., (2018), donde refieren que al conducir siguiendo una conducción ecológica se reduce el consumo de combustible en un $14 \%$ en comparación con los estilos de conducción normales.

Tabla 5: ANOVA para Consumo de Combustible por Modo de conducción

\begin{tabular}{|c|c|c|c|c|c|}
\hline Fuente & Suma de Cuadrados & Gl & Cuadrado Medio & Razón-F & Valor-P \\
\hline Entre grupos & 0,02916 & 1 & 0,02916 & 162,00 & 0,0000 \\
\hline Intra grupos & 0,00144 & 8 & 0,00018 & & \\
\hline Total (Corr.) & 0,0306 & 9 & & & \\
\hline
\end{tabular}

Tabla 6: Tratamientos para el análisis de diferencias significativas. Método: LSD (Consumo de combustible)

\begin{tabular}{|c|c|c|c|}
\hline Modo conducción & Casos & Media & Grupos Homogéneos \\
\hline 2 & 5 & 0,436 & $\mathrm{X}$ \\
\hline 1 & 5 & 0,544 & $\mathrm{X}$ \\
\hline
\end{tabular}




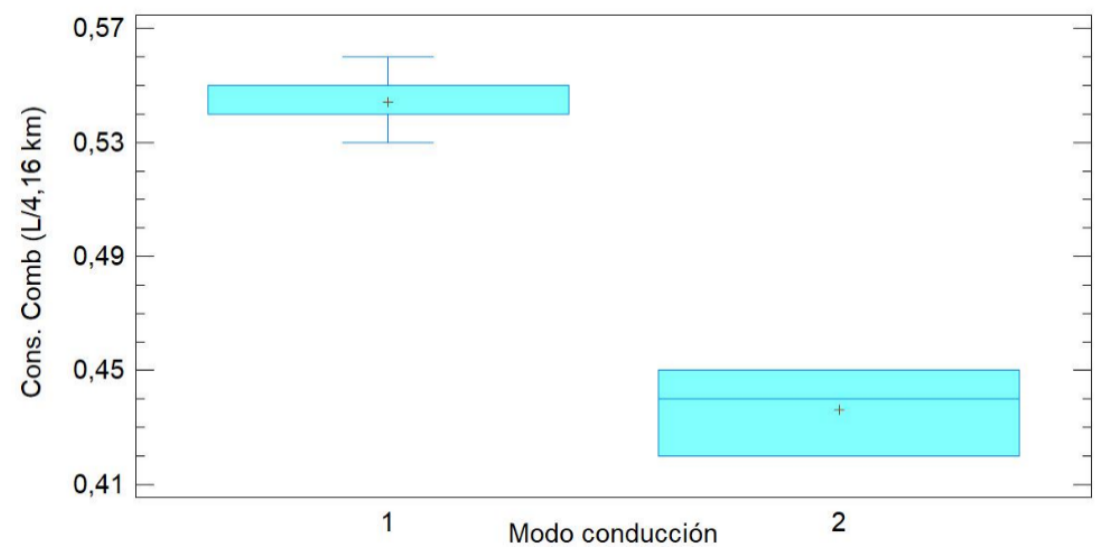

Fig. 3: Gráfico comparativo del consumo de combustible para los modos de conducción

Los resultados referentes al factor de emisión de $\mathrm{CO}$ se observar en la Figura 4 y Tabla 7, en la cual se aprecia que no existe diferencia significativa entre los modos de conducción, aunque la conducción eficiente experimenta cierto incremento. Los factores para el incremento del $\mathrm{CO}$ constituyen un alto par motor, el número de revoluciones y el coeficiente de aire (Gongbin y Chung, 2013), en el caso de la conducción eficiente se trata de mantener constantes las rpm y par motor medio, lo que indica que la variación de CO es mayor debido a que se tiene un coeficiente de aire de modo casi lineal con tendencia a mezcla rica. En trabajo desarrollado por Haworth y Symmons (2001) concluye que la conducción ecológica en modelación de ciclos de conducción no necesariamente produce reducción de $\mathrm{CO}$, porque se llevan a cabo en diferentes ambientes especificados de prueba y limitaciones, tales como tipo de vehículo, la hora del día, las rutas de conducción, etc., lo cual corrobora los datos obtenidos en el presente estudio.

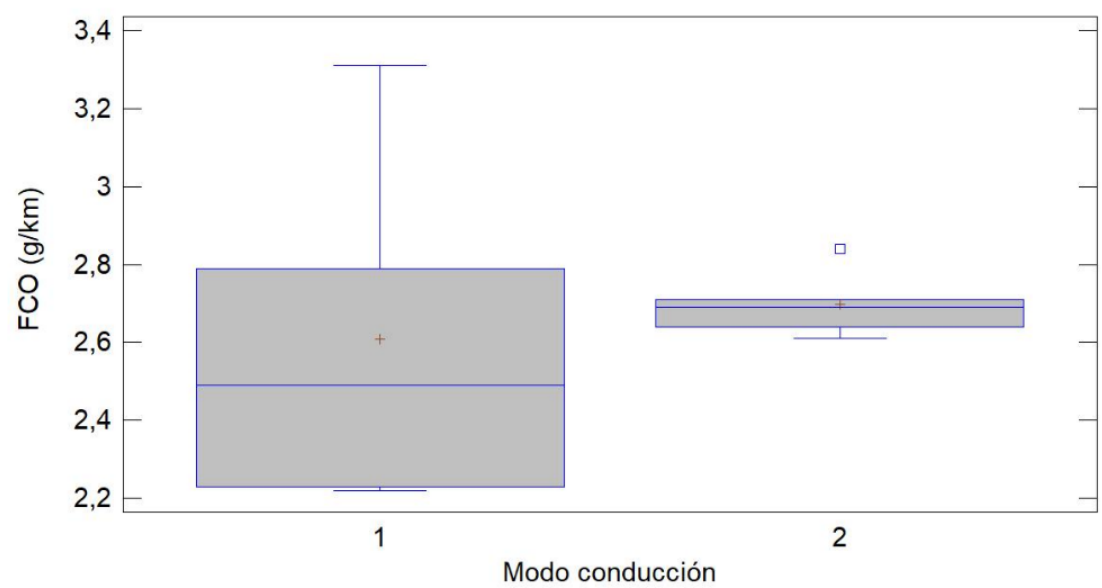

Fig. 4: Gráfico comparativo del factor de emisión del CO para los modos de conducción

Tabla 7: ANOVA para FCO por Modo de conducción

\begin{tabular}{|l|c|c|c|c|c|}
\hline \multicolumn{1}{|c|}{ Fuente } & Suma de Cuadrados & Gl & Cuadrado Medio & Razón-F & Valor-P \\
\hline Entre grupos & 0,02025 & 1 & 0,02025 & 0,19 & 0,6766 \\
\hline Intra grupos & 0,86476 & 8 & 0,108095 & & \\
\hline Total (Corr.) & 0,88501 & 9 & & & \\
\hline
\end{tabular}

En la Tabla 8 del ANOVA se observa que no existe diferencia significativa entre los modos de conducción para el caso del factor de emisión HC según el Valor-p, lo cual difiere de los estudios obtenidos por Mensing et al., (2014) donde se obtiene una reducción de los HC del 7.4 \% en una simulación en un vehículo liviano a una altura de 169 msnm aplicando el conducción eficiente, la diferencia de dichos resultados puede estar dada por las condiciones de altura a la cual se realizaron los estudios.

Tabla 8: Tabla ANOVA para FHC por Modo de conducción

\begin{tabular}{|l|c|c|c|c|c|}
\hline \multicolumn{1}{|c|}{ Fuente } & Suma de Cuadrados & Gl & Cuadrado Medio & Razón-F & Valor-P \\
\hline Entre grupos & 0,0010201 & 1 & 0,0010201 & 2,24 & 0,1728 \\
\hline Intra grupos & 0,0036428 & 8 & 0,00045535 & & \\
\hline Total (Corr.) & 0,0046629 & 9 & & & \\
\hline
\end{tabular}


En la Tabla 9 y Figura 5 se aprecia una reducción en el factor de emisión de NOx de $0.04 \mathrm{~g} / \mathrm{km}$ entre la conducción ecológica y la normal lo cual representa un 10 \% de reducción, motivado a que se trabaja a bajas rpm y el tiempo para la formación de NOx es menor, además la influencia del punto de encendido en esta condición perturba el equilibrio químico y disminuye la velocidad de reacción de la formación de NOx, estos resultados se corroboran con el estudio desarrollado por Haworth y Symmons (2001) donde se demostró que al aplicar conducción eficiente con una velocidad media entre $60 \mathrm{~km} / \mathrm{h}$ y $80 \mathrm{~km} / \mathrm{h}$ se reducen estas emisiones. debido a que el régimen de trabajo es más lineal y por tanto las temperaturas de trabajo en el interior del cilindro son más estables.

Tabla 9: Tratamientos para el análisis de diferencias significativas. Método: 95,0 porcentaje LSD (FNOx)

\begin{tabular}{|c|c|l|c|}
\hline Modo conducción & Casos & Media & Grupos Homogéneos \\
\hline 2 & 5 & 0,3938 & $\mathrm{X}$ \\
\hline 1 & 5 & 0,4344 & $\mathrm{X}$ \\
\hline
\end{tabular}

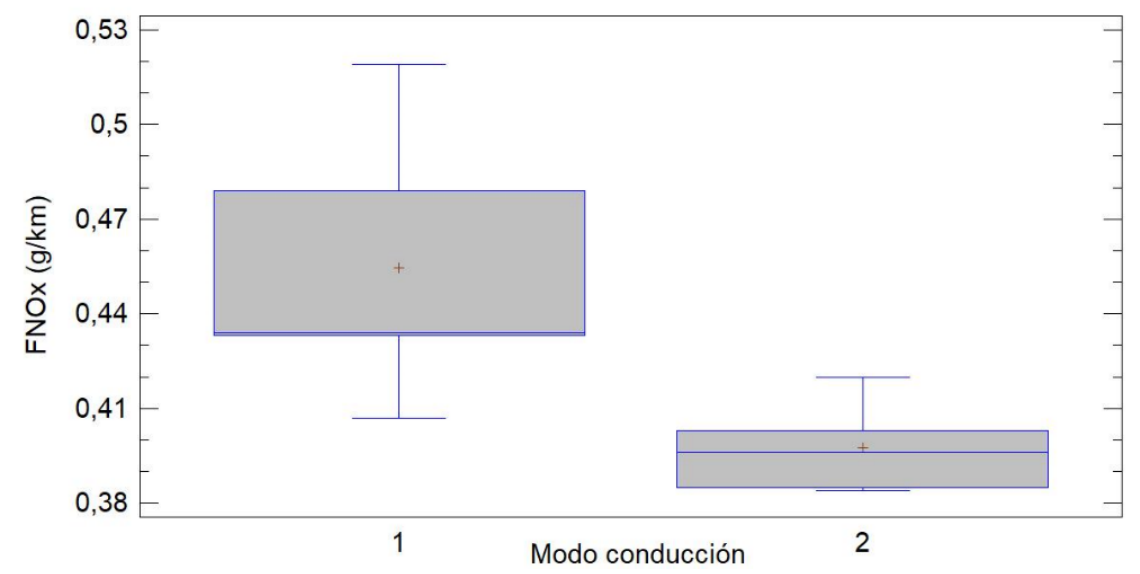

Fig.5: Gráfico comparativo del factor de emisión del NOx para los modos de conducción

\section{CONCLUSIONES}

De acuerdo con el estudio presentado, los resultados obtenidos y a la discusión sobre distintos aspectos del trabajo, se indican los efectos significativos que produce la técnica de manejo ecológico a una altura de 2810 msnm; con respecto al consumo de combustible y emisiones ( $\mathrm{CO}, \mathrm{HC}$ y NOx), destacándose los siguientes resultados: 1) La reducción del consumo de combustible al aplicar conducción eficiente en un dinamómetro de chasis es posible, obteniéndose un incremento en la autonomía del $19.8 \%$. 2) Los resultados muestran que las emisiones relacionadas con el $\mathrm{CO}$ y $\mathrm{HC}$ no muestran una diferencia significativa en función del modo de conducción. 3) La aplicación de técnicas de conducción eficiente reduce la cantidad de NOx emitidos por kilómetro con una reducción importante a pesar de que la altura influye directamente en la combustión. 4) Mediante la conducción ecológica se mejora la eficiencia de autonomía de un vehículo con los respectivos beneficios energéticos, ambientales y económicos.

\section{REFERENCIAS}

Rocha-Hoyos, J.C., E.A. Llanes-Cedeño, S. Celi-Ortega y D. Peralta-Zurita, Efecto de la Adicción de Biodiésel en el Rendimiento y la Opacidad de un Motor Diésel, Inf. Tecnol. 30(3), 137-146 (2019)

Guardia, Y., J. Márquez y otros cuatro autores, Mejoras A La Asignatura Diseño Estadístico De Experimentos Para Estudiantes De La Carrera De Ingeniería Mecánica, Revista ESPACIOS, 39(30), 10-26 (2018)

Huang, Y., E. G. Hong y otros cuatro autores, Eco-driving technology for sustainable road transport: A review. Renewable and Sustainable Energy Reviews, 93, 596-609 (2018)

Luján, J., C. Guardiola, B. Pla y A. Reig, Fuel and Pollutant Efficient Vehicle Speed Optimization in Real Driving Conditions. IFAC-PapersOnLine, 51(31), 225-232 (2018)

Rocha-Hoyos J., L. Tipanluisa, V. Zambrano, y A. Portilla, Estudio de un Motor a Gasolina en Condiciones de Altura con Mezclas de Aditivo Orgánico en el Combustible, Inf. Tecnol., 29(5), 325-334 (2018)

Vega, W.H., E.A. Llanes-Cedeño, J.V. Molina y J.C. Rocha-Hoyos, Revisión de las Características de Modelado y Optimización para el Diseño del Sistema de Suspensión Macpherson, Inf. Tecnol. 29(6), 221-233 (2018) 
Kolanjiappan, V., Reduction of Amine and Biological Antioxidants on Nox Emissions Powered by Mango Seed Biodiesel, doi: 10.17533/udea.redin.n84a06, Rev. Fac. Ing. Univ. Antioquia, (84), 46-54 (2017)

Quichimbla, F. y J.Solís, "Desarrollo de ciclos de conducción en ciudad, carretera y combinado para evaluar el rendimiento real del combustible de un vehículo con motor ciclo Otto en el Distrito Metropolitano de Quito," Escuela Politécnica Nacional, (2017).

Tipanluisa, L.E., A.P. Remache, C. R. Ayabaca y S.W. Reina, Emisiones Contaminantes de un Motor de Gasolina Funcionando a dos Cotas con Combustibles de dos Calidades, Inf. Tecnol. 28(1), 3-12 (2017)

Xu Y., H. Li y otros tres autores, Eco-driving for transit: An effective strategy to conserve fuel and emissions, Applied Energy, (194), 784-797 (2017)

Xuekai, C., K. Hong y L. Lu, A framework for estimating traffic emissions: The development of Passenger Car Emission Unit, Transportation Research Part D: Transport and Environment, 44, 78-92 (2016)

Ho, S. H., Y. D. Wong, y V. W. C. Chang, What Can Eco-driving Do for Sustainable Road Transport? Perspectives from a City (Singapore) Eco-driving Programme, Sustainable Cities and Society, 14, 82-88 (2015)

Liu, B., y C. Frey, Variability in Light-Duty Gasoline Vehicle Emission Factors from Trip-Based Real-World Measurements, Environ. Sci. Technol., 49(20), 12525-12534 (2015)

Qu, L., M. Li y otros cuatro autores, Multivariate Analysis Between Driving Condition And Vehicle Emission For Light Duty Gasoline Vehicles During Rush Hours, Atmospheric Environment, 110, 103-110 (2015)

Alam, M. S., y A. McNabola, A critical review and assessment of Eco-Driving policy \& technology: Benefits \& limitations, Transport Policy, 35 (2014)

Binder, S., G. Macfarlane, L. Garrow y M. Bierlaire, Associations among household characteristics, vehicle characteristics and emissions failures: An application of targeted marketing data, Transportation Research Part A: Policy and Practice, 59, 122-133 (2014)

Magaña, V. C., “Eco-driving: ahorro de energía basado en el comportamiento del conductor”, Carlos III de Madrid, (2014) Mensing, F., E. Bideaux, y otros tres autores, Eco-driving: An Economic or Ecologic Driving Style?, Transportation Research Part C: Emerging Technologies, (38), 110-121 (2014)

Krajzewicz D., M. Behrisch y P. Wagner, Second Generation of Pollutant Emission Models for SUMO, SUMO2014 - $2^{\mathrm{a}}$ Ed SUMO User Conference, 203-221, Berlin, Deutschland (2014)

Baric, D., G. Zovak y M. Periša, Effects of Eco-Drive Education on the Reduction of Fuel Consumption and CO2 Emissions, Promet - Traffic\&Transportation, 25(3), 265-272 (2013)

Gongbin, Q y E. Chung, Microscopic Simulation Study Of Eco-Driving Performance At Urban Intersections, $13^{\mathrm{a}}$ Ed Word Conference on Transport Research, 2-13, Rio de Janeiro, Brazil (2013)

Qiang, G. y E. Chung, Evaluating Effects of Eco-driving at Traffic Intersections Based on Traffic Micro-simulation, Australian Transport Research Forum 2011 Proceedings, Adelaide, Australia (2011)

Barlow, T., S. Latham, I. MacCrae, y P. Boulter, A reference book of driving cycles for use in the measurement of road vehicle emissions, Wokingham, (2009)

Barth, M., y K. Boriboonsomsin, Energy and Emissions Impacts of a Freeway-based Dynamic Eco-driving System, Transportation Research Part D: Transport and Environment, 14(6), 400-410 (2009)

Beusen, B., S. Broekx y otros cinco autores, Using on-board Logging Devices to Study the Longer-term Impact of an Ecodriving Course, Transportation Research Part D: Transport and Environment, 14(7), 514-520 (2009)

Rogula, W. Influence of Vehicular Traffic on Concentration and Particle Surface Composition of PM10 and PM 2.5 in Zabrze, Polish Journal of Envoirement, 17, 539-538 (2008)

Lapuerta, M., O. Armas, J. Agudelo, y C. Sanchez, Study of the Altitude Effect on Internal Combustion Engine Operation, Part 1: Performance. Inf. Tecnol., 17(5), 21-30 (2006)

Haworth N. y M. Symmons, The relationship between fuel economy and safety outcomes, Monash University Accident Research Centre (2001)

Pokharel, S., G. Bishop, y D. Stedman, Fuel-Based On-Road Motor Vehicle Emissions Inventory for the Denver Metropolitan Area, Denver: Department of Chemistry and Biochemistry (2001)

Frey, C., y D. Eichenberger, Remote sensing of mobile source air pollutant emissions: Variability and Uncertainty in OnRoad Emissions Estimates of Carbon Monoxide and Hydrocarbons for School and Transit Buses (1997) 
